\title{
Brain Machine Interface for Wrist Movement Using Robotic Arm
}

\author{
Sidhika Varshney *, Bhoomika Gaur *, Omar Farooq*, Yusuf Uzzaman Khan ** \\ * Department of Electronics Engineering, Zakir Hussain College of Engineering \& Technology, Aligarh, India. \\ ** Department of Electrical Engineering, Zakir Hussain College of Engineering \& Technology, Aligarh, India. \\ sidhika.varshney@gmail.com, bhoomika.gaur117@gmail.com,omarfarooq@amu.ac.in, yusufkhan1@gmail.com
}

\begin{abstract}
Brain Machine Interface (BMI) has made it possible for the disabled people to communicate with the external machine using their own senses. In the field of BMI, the invasive techniques have been widely used. This paper deals with the study of features of Electroencephalography (EEG), a non invasive technique that has been used for classifying two classes of movements, namely Extension and Flexion. Classification of movements is done on the basis of energy, entropy, skewness, kurtosis and their various combinations. The maximum accuracy of $91.93 \%$ has been obtained using discrete cosine transformation of energy and entropy. Finally the detected wrist movement is implemented on a mechanical Robotic Arm using ARDUINO UNO and MATLAB.
\end{abstract}

Keywords-EEG, interface, brain, invasive, non-invasive, signals

\section{INTRODUCTION}

There has been a sudden surge in the research for the Brain Machine Interface (BMI) technology as a mode of communication for the patients suffering from neurological disabilities. The discoverer of the existence of human EEG signals was Hans Berger (1873-1941) [1]. In 1926, Berger started to use the more powerful Siemens double coil galvanometer (attaining a sensitivity of $130 \mu \mathrm{V} / \mathrm{cm}$ ) [2]. The first report of 1929 by Berger included the alpha rhythm as the major component of the EEG signals, and the alpha blocking response [3]. Up to now, a lot of BCI systems have been developed for a variety of application purposes. For example, researchers at Graz university of technology have developed an EEG-based neuro-prosthesis by which a patient was able to grasp a simple object, and then release it after moving it from one place to another place [4], and a system integrated with functional electrical stimulation (FES) by which a tetraplegic patient could grasp a cylinder with the paralyzed hand through the control of beta oscillation signal [5]. Also complex robotic devices have been successfully and reliably controlled by BCIs, by exploiting smart interaction designs, such as shared control. Mill'an's group has pioneered the use of shared control in neuroprosthetics, by taking the continuous estimation of the user's intentions and providing appropriate assistance to execute tasks safely and reliably [6]. The basic principle for the working of BMI is the conversion of the neural signals into command for controlling external devices.
The acquisition of signals from brain can be in two ways Invasive or Non-invasive techniques.

Non-invasive technique works by using external sensors where as Invasive technique works by implanting sensors inside or superficially on the brain. Invasive technique has surgical procedures for implanting sensors which may cause medical problems and are permanently placed inside the brain.In this paper EEG, a Non invasive technique has been used for recording the brain signals. Among the many invasive and noninvasive brain signal acquisition techniques, Electroencephalogram (EEG) is the most commonly adopted non-invasive method in BCI because of its high temporal resolution, ease of use, low cost and portability [7]. This paper deals with classification of two classes of movements that is Flexion and extension as shown in Figure 1. The data is used to classify the movements.
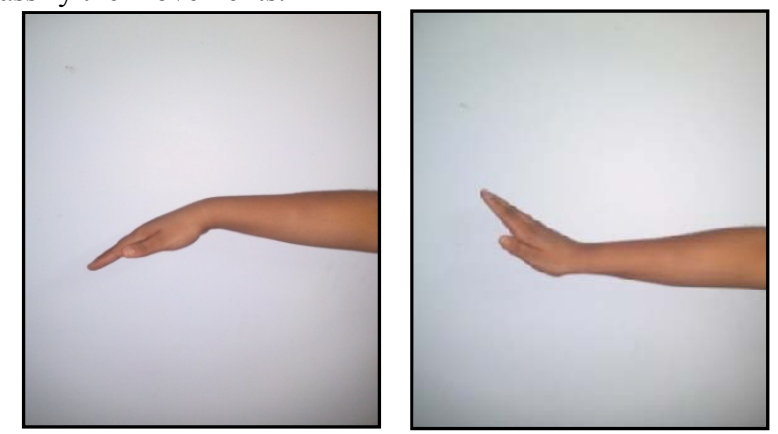

Figure 1. Flexion and extension wrist movements respectively

The final result has been demonstrated on the Robotic Arm using ARDUINO UNO board.

\section{ELECTROENCEPHALOGRAPHY (EEG)}

The central nervous system (CNS) comprises of nerve cells and glia cells that are located between neurons. These neurons communicate with each other by sending impulses through synaptic activities.

During such synaptic activities, there is flow of cat-ions from the nerve cell, or an inflow of an-ions into the nerve cell. This flow ultimately causes a change in potential along the nerve cell membrane. The portion of these currents that flow through the extracellular space is directly responsible for the generation of 
field potentials. These field potentials, usually with less than $100 \mathrm{~Hz}$ frequency, are called EEGs.

There are mainly four kinds of brain waves distinguished by their different frequency ranges. These frequency bands from low frequencies to high frequencies are called alpha $(\alpha)$, theta $(\theta)$, beta $(\beta)$ and delta $(\delta)$ [3]. Many brain disorders are diagnosed by visual inspection of EEG signals. In healthy adults, the amplitudes and frequencies of such signals vary from one state of a human to another, such as wakefulness and sleep. The characteristics of the waves also vary with age. Table I presents the comparison of various EEG bands, the associated brain waves and their state of occurrence.

TABLE I. COMPARISON OF EEG BANDS

\begin{tabular}{|c|c|l|}
\hline Type & Frequency (Hz) & \multicolumn{1}{|c|}{ State of Occurrence } \\
\hline Delta & $0.5-4$ & $\begin{array}{l}\text { a) adults slow wave sleep } \\
\text { b) in babies }\end{array}$ \\
\hline Theta & $4-8$ & $\begin{array}{l}\text { a) young children } \\
\text { b) drowsiness or arousal } \\
\text { in older children and } \\
\text { adults } \\
\text { c) idling }\end{array}$ \\
\hline Alpha & $8-13$ & $\begin{array}{l}\text { a) relaxed state } \\
\text { b) eye blinking }\end{array}$ \\
\hline Beta & $13-40$ & $\begin{array}{l}\text { a) alert/working } \\
\text { b) active, busy or anxious } \\
\text { thinking }\end{array}$ \\
\hline
\end{tabular}

\section{OVERALL BMI SETUP}

Figure 2 shows the block diagram of a typical Brain Machine Interface. It consists of a data acquisition block, a signal processing block, feature extractor, a classifier and an actuator. Additionally a feedback arrangement may also be used.

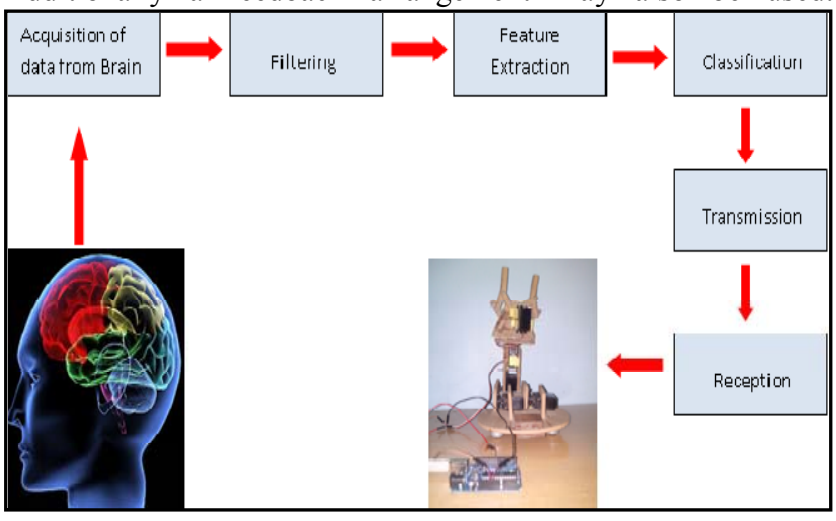

Figure 2. Block Diagram of Brain Machine Interface

The task of data extractor is to collect the electrophysiological signals from the brain and transfer them to computer in suitable form. The signals obtained are generally amplified by the recording system. Next, the signal processing block removes excessive noise including the line frequency of
$50 \mathrm{~Hz}$, thus improving the signal to noise ratio. Further signal processing may include spike detection and analysis. Next, the feature extractor calculates various signal features including energy, entropy, skewness, kurtosis and discrete cosine transform.

These features then form the basis for the classification of movements by the classifier. Finally, the classifier output or the result of detection process is transferred to an actuator e.g. a robotic arm which imitates the required movement. The feedback block, if used, sends error signals back to the brain so as to improve the accuracy of the system.

\section{A. Data Acquisition}

The experimental setup for the recording of EEG signals is shown in Figure 3. The EEG recording facility was set up in the signal processing laboratory of Electronics Engineering Department, Z.H.C.E.T., A.M.U. Data was recorded using a Brain Tech clarity system software version 3.4, hardware version 1.4. Firstly, the electrodes were placed over the scalp using the gel to keep the contact impedance within the limit and reduce the noise effects. The maximum allowed contact impedance was set at 50ohms. For placing electrodes the standard 10-20 system was used. According to this system,

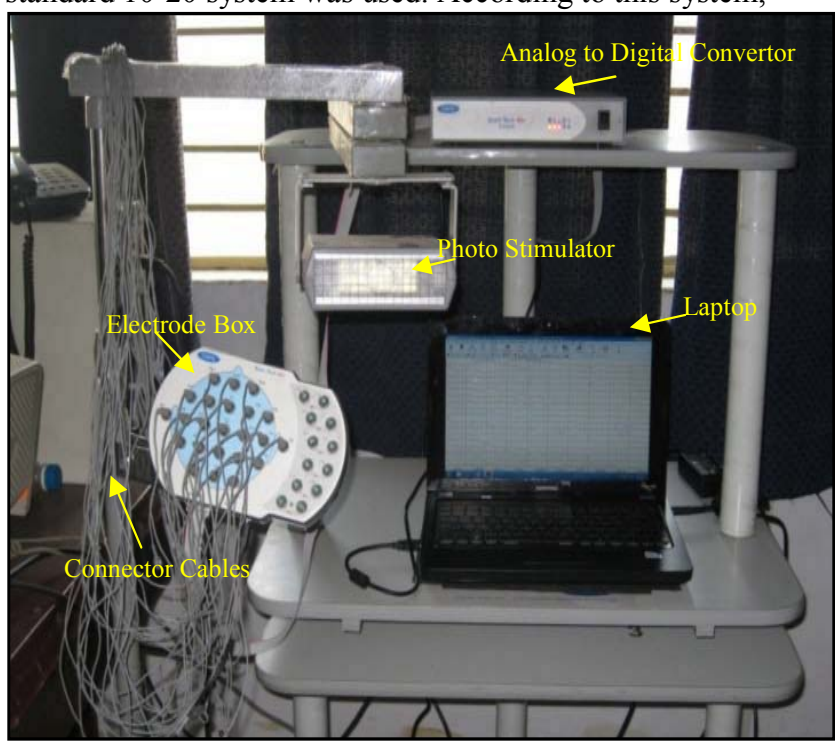

Figure 3. Experimental Setup for data acquisition.[8]

the EEG electrodes are normally distributed on the scalp with the actual distances between adjacent electrodes being $10 \%$ or $20 \%$ of the total front-back or right-left distance of the skull [9]. Also, a minimum of 25 electrode inputs ( 21 on the scalp, one for the system reference, one for ground, and 2 extra) are recommended.

The EEG was recorded for two wrist movements of left hand namely, flexion and extension. Each movement was recorded by sixteen channels for duration of three seconds. A total of eighteen trials were used for each movement. The sampling frequency was chosen as $256 \mathrm{~Hz}$, thus, yielding a signal length of 768 samples. The EEG waveforms of the two wrist movements are shown in Figure 4. 


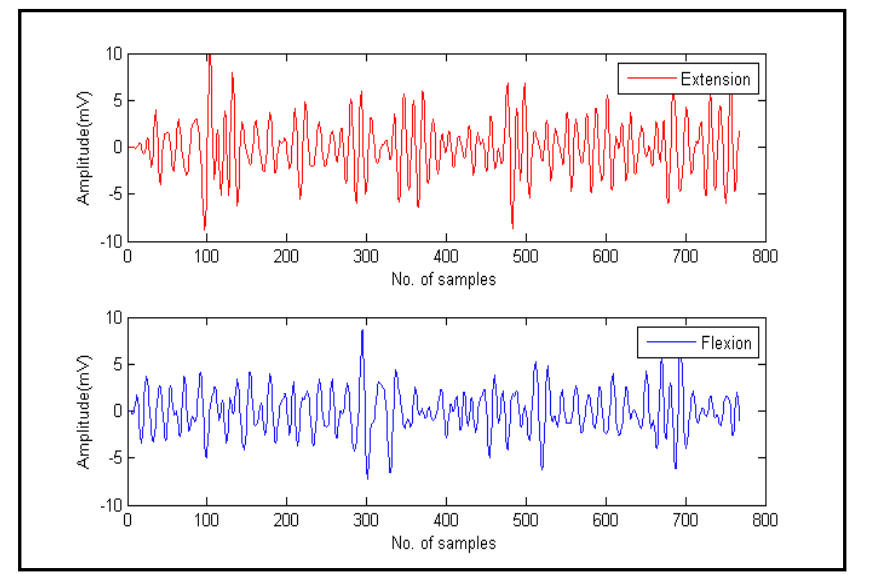

Figure 4. EEG signals corresponding to flexion and extension

\section{B. Signal Processing}

The EEG signals are generally affected by sinusoidal disturbance from ac power supply having a frequency of $50 \mathrm{~Hz}$. For removing this noise, a band pass filter (Butterworth, $7^{\text {th }}$ order) with cutoff frequencies $10 \mathrm{~Hz}$ and $30 \mathrm{~Hz}$ has been designed in MATLAB (R2012a). In this way the dc component is also removed. With the frequency range of $10-30 \mathrm{~Hz}$ only the most informative alpha and beta waves remain in the signals which together dominate during thinking, attention, focus and other brain activities. Figure 5 shows the plots of frequency spectrum of raw EEG signal and filtered.

\section{Feature Extraction}

The filtered data for each movement was obtained in the form a $768 \times 16$ matrix with each signal having 768 samples and viewed by 16 channels. The energy and entropy of each signal were calculated using equations (1) and (2) respectively. Also the kurtosis and skewness were calculated using equations (3) and (4) respectively.

To reduce the complexity discrete cosine transform of the above features was taken and only first four coefficients (except the first) were retained. Finally equations (5) and (6) were used for the calculation of discrete cosine transform coefficients.

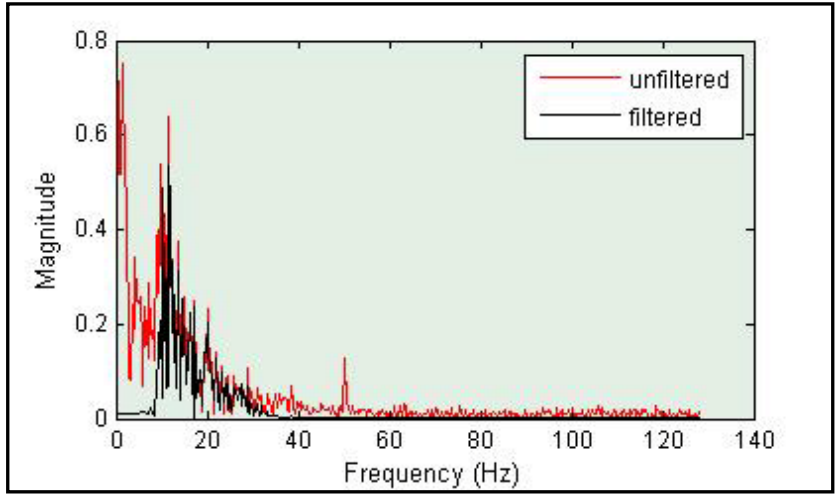

Figure 5. Fourier Transform of Raw and Filtered data.

$$
\begin{gathered}
e=\sum_{i=1}^{N} X_{i}^{2} \\
H=-\sum_{i=1}^{N}\left(X_{i} * \log \left(X_{i}^{2}\right)\right) \\
k=\frac{E(X-\mu)^{4}}{\sigma^{4}} \\
s=\frac{E(X-\mu)^{3}}{\sigma^{3}} \\
y(k)=w(k) \sum_{i=1}^{N} F(i) \cos \frac{\pi(2 i-1)(k-1)}{2 N} \\
w(k)=\left(\begin{array}{l}
\frac{1}{\sqrt{N}}, \quad k=1 \\
\frac{\sqrt{2}}{\sqrt{N}}, \quad 2 \leq k \leq N
\end{array}\right)
\end{gathered}
$$

Where, $e=$ energy of the signal, $H=$ entropy of the signal,

$F(i)=\mathrm{i}^{\text {th }}$ sample of feature $\mathrm{F}$.

$y(k)=\mathrm{k}^{\text {th }}$ coefficient of discrete cosine transform,

$X(i)=\mathrm{i}^{\text {th }}$ sample amplitude of EEG signal,

$k=$ kurtosis of the EEG signal

$s=$ skewness of the EEG signal

$E(x)=$ Expected value of some variable $x$

$\mu=$ mean of signal

$\sigma=$ standard deviation of signal

$N=$ total number of samples $(\mathrm{N}=768)$.

\section{Classification}

The classification was performed by randomly selecting four trials of each movement as test data and the remaining fourteen trials as train data. In most of the cases diagonal linear and diagonal quadratic models of classifiers have been used as the best results were obtained from them. The classification was performed thousand times for each feature and classifier model. Finally, the average of obtained accuracies was calculated.

\section{RESULT}

Table II presents the average accuracies obtained with various features and classifier models. The accuracies are very much dependent on selection of features and classifier model. The best results are obtained using discrete cosine transform. The data used in this study has been collected from one subject only. That is why the classification results are less accurate. If more subjects are involved then fairly high accuracies can be obtained.

However, the average accuracies obtained in this analysis are acceptable and hence the output of this analysis can be efficiently implemented over a control device.

\section{INTERFACING WITH ROBOTIC ARM}

The result with maximum accuracy of classification has been implemented on a Robotic arm via ARDUINO UNO board. Figure 6 shows the complete setup of interfacing. MATLAB (R2012a) is interfaced with ARDUINO UNO using 
TABLE II. ACCuracies ObTAined Using VARIOUS FeAtures AND Classifier Model

\begin{tabular}{|c|c|c|c|}
\hline S. No. & Features & Classifier Model & Percentage Accuracy \\
\hline \multirow{2}{*}{1} & \multirow{2}{*}{ Energy, Entropy, Skewness } & Linear & 81.08 \\
\hline & & Quadratic & 87.34 \\
\hline \multirow{2}{*}{2} & \multirow{2}{*}{ Energy, Entropy, Kurtosis } & Linear & 82.85 \\
\hline & & Quadratic & 84.18 \\
\hline \multirow{2}{*}{3} & \multirow{2}{*}{ Energy, Entropy, Skewness, Kurtosis } & Linear & 83.62 \\
\hline & & Quadratic & 82.88 \\
\hline 4 & Energy and Kurtosis(with Discrete Cosine Transform) & Linear & 84.60 \\
\hline 5 & Entropy and Kurtosis(with Discrete Cosine Transform) & Linear & 79.59 \\
\hline 6 & Energy and Skewness(with Discrete Cosine Transform) & Linear & 82.03 \\
\hline 7 & Energy and Skewness(with Discrete Cosine Transform) & Linear & 79.80 \\
\hline \multirow[b]{2}{*}{8} & \multirow{2}{*}{ Energy, Entropy and Skewness (with Discrete Cosine Transform) } & Linear & 89.66 \\
\hline & & Quadratic & 73.89 \\
\hline \multirow[b]{2}{*}{9} & \multirow{2}{*}{ Energy, Entropy and Skewness (with Discrete Cosine Transform) } & Linear & 88.40 \\
\hline & & Quadratic & 71.40 \\
\hline 10 & $\begin{array}{l}\text { Energy, Entropy, Kurtosis and Skewness (with Discrete Cosine } \\
\text { Transform) }\end{array}$ & Linear & 85.51 \\
\hline 11 & Discrete Cosine Transform & Linear & 91.93 \\
\hline
\end{tabular}

MATLAB support package for ARDUINO (ARDUINO IO package). GUI window is used as mode for the selection of Wrist movement i.e. Flexion or extension. On pressing either of the buttons out of 36 trials of recordings one trial is randomly selected and is used as test data. The remaining 35 trials are used to train the classifier which then classify the test data and return the output to the ARDUINO UNO through USB cable. The final detected movement is then performed on the robotic

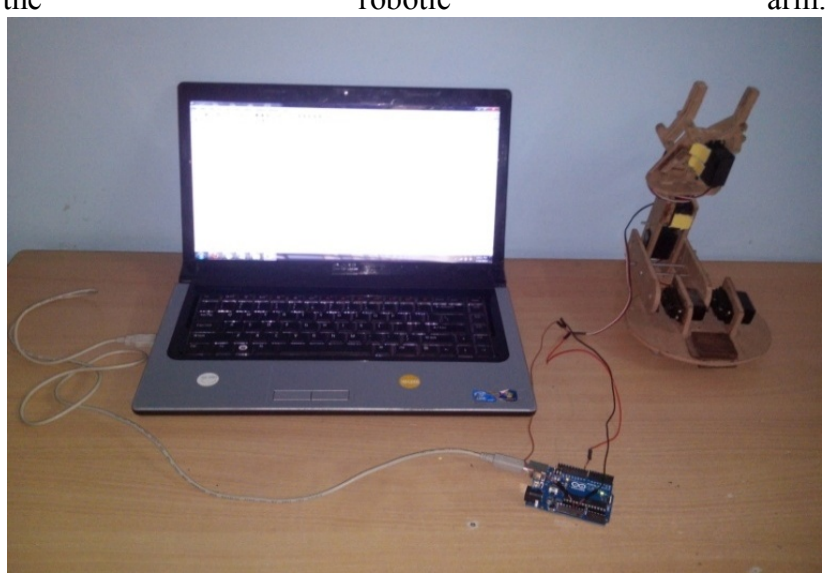

Figure 6. Setup for interfacing Robotic Arm

\section{CONCLUSION}

The study reveals that this analysis can be used to distinguish more classes of movements. However, this may require the use of more features to get acceptable results. Additionally, segmentation or wavelet based approach can yield better results. Similarly, improved classifier models can be designed for better recognition. If this work is carried further then an independent Brain Machine Interface can be developed.

\section{ACKNOWLEDGMENT}

This work was supported by the Department of Electronics Engineering, ZH College of Engineering and Technology, AMU, Aligarh INDIA.

\section{REFERENCES}

[1] A. Massimo, "In Memoriam Pierre Gloor (1923-2003): an appreciation", Epilepsia, 45(7), 882, July 2004.

[2] A. M. Grass, and F. A. Gibbs, "A Fourier transform of the electroencephalogram”, J. Neurophysiol., 1, 521-526, 1938.

[3] S.Sanei and J. Chambers, "EEG Signal Processing," John Wiley \& Sons, Ltd, 2007, pp 4-13. 
[4] G. Pfurtscheller, and R. Rupp, "EEG-based neuroprosthesis control: a step towards clinical practice", Neuroscience letters, vol.382, no.1, pp.169-174, 2005.

[5] G. Pfurtscheller, G.R. Muller, J. Pfurtscheller, H.J. Gerner, and R. Rupp, " 'thought'-control of functional electrical stimulation to restore hand grasp in a patient with tetraplegia", Neuroscience letters, vol.351, no.1, pp.33-36, 2003.

[6] T. Carlson, L. Tonin, S. Perdikis, R. Leeb, and J. R. Mill'an, "A Hybrid BCI for Enhanced Control of a Telepresence Robot", Proceedings 35th Annual International Conference of the IEEE EMBS, Osaka, Japan, July 2013.
[7] X. Jiang, T. Cao, F. Wan, P. U. Mak, P. Mak, and M. I. Vai, "Implementation of SSVEP based BCI with Emotiv EPOC," Proceeding IEEE International Conference on Virtual Environments,HumanComputer Interfaces and Measurement Systems, Tianjin, China, pp. 3437, July 2012

[8] P. Sharma, "Automatic detection of non-convulsive seizures," M. Tech dissertation, Dept. Electronics Engineering, Aligarh Muslim Univ., Aligarh, India, 2012.

[9] Thasneem Fathima, M. Bedeeuzzaman, Omar Farooq and Yusuf U Khan, "Wavelet Based Features for Epileptic Seizure Detection", MES Journal of Technology and Management, pp. 108-112, May 2011. 